\title{
SISTEMA DE COMUNICACIÓN INDUSTRIAL PARA EL MONITOREO DE LAS ESTACIONES DE AGUA POTABLE EN CIENEGUILLA-LIMA
}

\author{
Industrial communication system for monitoring stations \\ in drinking water Cieneguilla-Lima
}

\author{
Omar Freddy Chamorro Atalaya ${ }^{1}$ \\ Margarita Fredesvinda Murillo Manrique ${ }^{2}$
}

\section{RESUMEN}

El presente artículo está dirigido a determinar si es posible desarrollar un sistema de comunicación entre las estaciones de agua potable en el distrito de Cieneguilla, ya que no cuentan con uno. De esa manera, se logrará un sistema de monitoreo que cumpla con estándares internacionales y nacionales. Por lo tanto, las estaciones remotas (ER) hidráulicamente dependientes se comunicarán entre sí y la información de cada ER será llevada y visualizada en el SCADA de La Atarjea. Para ello, se investiga de qué manera los parámetros del sistema de comunicación influyen en la calidad del de monitoreo. La recolección de los datos se realizó con un formulario válido en su contenido, el cual fue analizado con estadística inferencial, específicamente, con el chi cuadrado, utilizando tablas de valores obtenidos y esperados. Los resultados indicaron que los parámetros del sistema de comunicación influyen positivamente en el desarrollo de un sistema de monitoreo, lo que garantiza valores óptimos de calidad de comunicación. Esto es, a partir de la utilización correcta de valores de ganancia de las antenas y de la potencia del trasmisor, se aseguran valores óptimos de potencia de recepción, margen de desvanecimiento y relación señal a ruido.

Palabras clave: Estaciones remotas, SCADA, calidad del sistema de comunicación, potencia de recepción, margen de desvanecimiento, relación señal a ruido.

\begin{abstract}
This article is aimed at determining the feasibility of developing a communication system Drinking water stations in the district of Cieneguilla; for a monitoring system that complies with international and national standards, so that the hydraulically dependent remote stations (ER) to communicate with each other and also for the information of each ER is taken and displayed on the SCADA Atarjea. For this we investigate how the system parameters affect communication quality monitoring system.

The data collection was performed with a valid form in its content, which was analyzed with inferential statistics specifically for the Chi Square values obtained using tables and expected. The results indicated that the parameters of the communication system positively influence the development of a monitoring system, ensuring optimal values of communication quality. That is, from the proper use of gain values of the antennas and transmitter power, optimal values of power reception, fading margin and Signal to Noise is guaranteed.
\end{abstract}

Keywords: Remote stations, SCADA, Quality communication system, Front power, fade margin, Signal to Noise.

1 Ing. Electrónico Colegiado. Mg. en Ingeniería de Sistemas. Docente de posgrado en la Universidad Nacional del Callao en asignaturas de Automatización y Control de Procesos. Director de la Escuela Profesional de Ingeniería Mecánica y Eléctrica de la Universidad Nacional Tecnológica de Lima Sur -UNTELS. Docente en la UNTELS. E-mail: omar_chamorro1@hotmail.com

2 Ing. Electricista Colegiada con CIP 59410. Mg. en Docencia y Gestión Educativa. Dra. en Educación. Certificación de Doctora Honoris Causa de la UNAP. Bachiller en Ing. Eléctrica y Electrónica. Decana de la Facultad de Ingeniería y Gestión de la Universidad Nacional Tecnológica de Lima Sur -UNTELS. Docente Metodóloga en el Programa TITES de la FI-URP. Docente Ordinario de la UNTELS. Docente de la Escuela Profesional de Ingeniería Mecatrónica de la URP E-mail: margaritamurillom@yahoo.es 


\section{INTRODUCCIÓN}

Un aspecto que se debe considerar en el rendimiento y la disponibilidad de un proceso es el que se refiere a las fallas en los equipos y en el mantenimiento, las cuales cuestan tiempo y dinero. Aunque al principio se crea que son problemas poco significativos, si no se detectan, pueden aumentar y causar dańos importantes. De esa manera, las tareas más simples de mantenimiento se convierten en reparaciones mayores que, incluso, pueden llevarnos a una parada de planta no programada.

Este sistema de comunicación se desarrolla para trabajar en frecuencias no licenciadas $(900 \mathrm{Mhz}$, $2.4 \mathrm{GHz}$, y $5.8 \mathrm{GHz}$ ). Es decir, no se requiere ningún trámite de autorización para su utilización. En esta investigación, se utilizó la frecuencia de $5.8 \mathrm{GHz}$, con la finalidad de no contravenir el cumplimiento de la norma del Ministerio de Transportes y Comunicaciones (MTC) respecto al nivel de potencia isotrópica efectiva radiada, para estaciones cuya transmisión se realiza en frecuencias no licenciadas. En ese sentido, se desarrolló este sistema de monitoreo buscando no exceder el valor de 36 $\mathrm{dBm}$. Al desarrollar esta investigación, se determinó si, a partir de las ubicaciones actuales de las ER, es posible establecer la comunicación, puesto que, en la banda de frecuencia en la que se transmite, se requiere una línea de vista entre las estaciones de transmisión y recepción.

\section{MATERIAL Y MÉTODOS}

Se realizó un análisis respecto de la información de proyectos asociados que optimizan el uso del agua como recurso fundamental para la vida y su relación con la tecnología actual. Se presenta el estado de arte respecto a la importancia del desarrollo de un sistema de monitoreo telemétrico entre estaciones de agua potable para el distrito de Cieneguilla.

\section{CARACTERIZACIÓN DEL PROBLEMA DE INVESTIGACIÓN}

$\mathrm{Al}$ respecto de los cortes de agua, Cabrera, en su libro El suministro de agua urbano en España, afirma que:

La prueba más palmaria de las deficiencias de los sistemas de distribución de agua es la predisposición de los responsables de turno a interrumpir el suministro tan pronto en las fuentes de suministro las reservas disponibles disminuyen, hasta alcanzar el nivel de alerta. Por esa razón, cortar el agua supone incomodar a la población, sin garantía de que esta reduzca su consumo. La necesidad la obliga a almacenarla en recipientes, bañeras y cubos, y una parte de ella no se va a utilizar después, lo que genera la pérdida de este recurso. Los cortes de agua son justificados por una demagogia establecida y así al usuario, desconocedor de que el verdadero problema está en la deficiencia de la red, se le presenta la solución del corte como la única respuesta posible a la falta de solidez de las fuentes de suministro que abastecen a la ciudad. [1].

En el mismo sentido, García, en su libro titulado Problemática de los abastecimientos urbanos, señala que «La respuesta posible a la falta de agua en las zonas urbanas, es debido a una mala administración de los elementos que componen la gestión del suministro de agua potable» [2]. Más adelante, añade lo siguiente:

Para gestionar la demanda y mejorar la garantía de suministro están los planes de gestión ante fallas, estas se apoyan en tres pilares básicos: Alimentar el sistema con fuentes de suministro de la mayor fia- 
bilidad, disponer de redes estancas que minimicen las pérdidas y administrar la demanda gestionando juiciosamente los recursos; por lo tanto, equilibrando estas tres estrategias, complementarias entre sí, se aseguraría un abastecimiento óptimo del agua para las zonas urbanas [2].

La Organización Mundial de la Salud, en su Guia para la calidad del agua potable, establece que «La evaluación del sistema de abastecimiento de agua potable es igualmente aplicable a grandes instalaciones con redes de distribución de agua por tuberías, incluidas las bombas manuales y los sistemas de abastecimiento domésticos particulares» [3].

A partir de estas fuentes, se pueden evaluar infraestructuras existentes, o planes para la instalación de nuevos sistemas de abastecimiento o la mejora de los existentes. Puesto que la calidad del agua potable varía de unos puntos a otros, en el mismo sistema, el objetivo de la evaluación debe ser determinar si el agua potable suministrada llegará eficientemente al consumidor.

Respecto a los gastos económicos para el usuario y la problemática del medio ambiente que se debe considerar, Ruiz señala lo siguiente:

...es de mucha importancia el uso eficiente del agua porque está relacionado con los factores de gasto económico y con el medio ambiente. Por ejemplo, dentro de las empresas el agua también supone un coste directo y real donde el derroche de la misma, conlleva a un factor de gasto económico que crece de manera importante a tener en cuenta todo lo que de una manera indirecta aumenta dicha cifra. Por lo tanto, debemos perseguir unas instalaciones sostenibles y respetuosas, no solo desde el punto de vista económico, mejorando la cuenta de resultados, sino desde el compromiso que todos debemos mantener con el medio ambiente. [4]

Esteban, en su libro Eficiencia del agua en las Ciudades, otorga especial énfasis al siguiente aspecto:

...se dispone de muchas opciones cuando se habla de ahorrar agua y energía, y esto ha de hacerse considerando varios factores desde la optimización de las facturas, pasando por la formación del personal y considerando los proyectos en su fase de diseño, a la realización de estudios y eco-auditorias de Hidroeficiencia. . [5]

Por lo tanto, de los autores anteriormente citados, podemos señalar la relación directa que existe entre el consumo de agua y la demanda de energía, ya que ahorrar un litro de agua en nuestros establecimientos supone no solo el ahorro del litro en sí mismo, sino también de los gastos derivados de su utilización, como el bombeo, el calentamiento, la acumulación, la recirculación, las tasas de alcantarillado, la depuración y el control del nivel de agua, que son procesos que requieren de energía eléctrica.

El problema del agua en nuestro país viene siendo crítico desde años atrás. Al respecto, es importante indicar que la UNICEF, en su estudio Agua y saneamiento en el Perú, señala que

...en el sector de agua potable y saneamiento del Perú, se han logrado importantes avances en las últimas dos décadas del siglo XX y primera del siglo XXI, como el aumento del acceso de agua potable del $30 \%$ al $62 \%$ ocurrido entre los ańos 1980 al 2004 y el incremento del acceso de saneamiento del $9 \%$ al 30\% entre los años 1985 al 2004 en las áreas rurales. [6]

Es conveniente mencionar que la oferta de agua en el Perú y en el mundo está cada vez más limitada y la demanda sigue en aumento. Por ello, suministrar y manejar el agua en todos los sectores es importante y se logrará mediante políticas públicas concertadas, además de instituciones fortalecidas 
Esto permite establecer que quedan muchos retos en el sector, tales como la insuficiente cobertura de servicios, la mala calidad de la prestación de estos que pone en riesgo la salud de la población y la deficiente sostenibilidad de los sistemas construidos.

El Ministerio de Vivienda Construcción y Saneamiento del Perú en su D.S No 007-2006 R.VM. Nº02-2007-VIVIENDA-VMCS, en el Plan nacional de saneamiento 2007-2015, señala lo que sigue:

En el año 2004, la población total del Perú fue de 27.5 millones de habitantes, de los cuales el 71\% residió en el área urbana y el $29 \%$ en el área rural. La cobertura de servicios de agua potable fue de $76 \%$ y saneamiento de $57 \%$. En lo que respecta, a las áreas urbanas la cobertura fue de $81 \%$ en agua y $68 \%$ en saneamiento. Por otro lado, en las áreas rurales, donde vive un $27 \%$ de la población, la cobertura de agua potable fue de $62 \%$ y saneamiento de $30 \%$. [7]

Como podemos observar, el acceso al agua potable es fundamental para la salud, uno de los derechos humanos básicos y un componente de las políticas eficaces de protección de la salud; sin embargo, en la actualidad, existen todavía algunos distritos en la ciudad de Lima donde el abastecimiento de agua, a causa del suministro, es un problema muy serio a tener en cuenta en el corto plazo.

\section{CONSIDERACIONES GENERALES PARA EL DESARROLLO DEL SISTEMA DE MONITOREO}

Los alcances generales para el desarrollo del sistema de monitoreo comprenden: a) el estudio de campo electromagnético con el analizador de espectros en la zona de Cieneguilla, este instrumento permite determinar la banda de frecuencia libre menos congestionada; b) el diseńo del sistema de comunicación local entre estaciones remotas; c) diseño del sistema de comunicación principal SCADA, ubicado en la Atarjea; y, finalmente, el análisis de los factores de transmisión en los parámetros de calidad del sistema de monitoreo. A continuación, detallamos cada uno de los puntos mencionados.

a. En cuanto al estudio de campo electromagnético: se verificó a través de pruebas con el analizador de espectros en la zona de ubicación de las estaciones remotas, en las bandas de $900 \mathrm{MHz}$, 2.4GHz y 5.8 GHz. La que se utilizó garantiza únicamente la transmisión de data de telemetría de las estaciones remotas.

b. En cuanto al diseño del sistema de comunicaciones local entre las estaciones: teniendo como referencia las ubicaciones geográficas (latitud, longitud, altitud) de cada estación y según las recomendaciones de Caprile [8] sobre el desarrollo de aplicaciones con comunicaciones remotas, se procedió a realizar los estudios de los perfiles de cada uno de los enlaces entre las estaciones. De ese modo, se garantiza la línea de vista entre estas. La topología de telecomunicaciones local incluye la topología del SCL: estrella extendida.

c. En cuanto al diseño del sistema de comunicación principal SCADA: este diseño se justifica en la teoría de Blake [9] sobre sistemas electrónicos de comunicaciones, así se realizó el estudio de perfiles de los enlaces que permitirán transmitir la señal de telemetría hasta la estación principal, ubicada en la Atarjea. La topología de telecomunicaciones principal incluye la que es redundante $1+1$. 
TABLA 1. TERMINOLOGÍA USADA EN EL DESARROLLO DEL SISTEMA DE MONITOREO, 2017.

\begin{tabular}{|c|c|}
\hline Ancho de banda & $\begin{array}{l}\text { Cantidad de información o datos que se puede enviar por un medio de comunicación en un } \\
\text { tiempo determinado. Se mide en bits por segundo (bps). }\end{array}$ \\
\hline ER & Estación remota \\
\hline Frecuencia libre & $\begin{array}{l}\text { Son las frecuencias autorizadas por el MTC según Resolución Ministerial № 777-2005-MTC/03 } \\
\text { Estas son: } \\
\text { - Banda de } 900 \mathrm{MHz}(902 \mathrm{MHz}-928 \mathrm{MHz}) ; \\
\text { - Banda de } 2.4 \mathrm{GHz}(2400 \mathrm{MHz}-2483.5 \mathrm{MHz}) ; \\
\text { - Banda de } 5.8 \mathrm{GHz}(5725 \mathrm{MHz}-5850 \mathrm{MHz}) \text {. }\end{array}$ \\
\hline FHSS & Modulación basada en el salto de frecuencias. \\
\hline Redundancia & $\begin{array}{l}\text { Este aspecto se entiende como el medio alterno que se utiliza para comunicar dos puntos } \\
\text { remotos. }\end{array}$ \\
\hline $\begin{array}{l}\text { Sistema de comunicación local } \\
\qquad(\mathrm{SCL})\end{array}$ & $\begin{array}{l}\text { Este consiste en la comunicación entre estaciones remotas y en permitir que la geografía } \\
\text { estos enlaces coincidan con las dependencias hidráulicas. }\end{array}$ \\
\hline $\begin{array}{l}\text { Sistema de comunicación } \\
\text { principal }(S C P)\end{array}$ & $\begin{array}{l}\text { Se define como el sistema de comunicaciones principal al enlace primordial con la red de } \\
\text { Sedapal. }\end{array}$ \\
\hline
\end{tabular}

\section{TOPOLOGÍA DE COMUNICACIONES}

Se analizaron y definieron los enlaces de radio de tipo local, los cuales están agrupados en cuatro subsistemas. Así mismo, se analizaron los enlaces de radio principales, los mismos que permitirán enlazar estas cuatro estaciones con la principal, de acuerdo con las condiciones de ubicación geográfica y con las alturas de las torres ventadas o postes de concreto, se consideró los modelos de Apaza redes de abastecimiento de agua potable [10].

\subsection{Descripción}

El sistema de agua potable y de alcantarillado del esquema Cieneguilla está formado por cuatro subsistemas que contienen 35 estaciones remotas, distribuidas de la siguiente forma, tal como se indica en SEDAPAL [11]:

El subsistema No 1: contiene 10 estaciones, divididas de la siguiente forma:

- Reservorios proyectados (RP): 2

- $\quad$ Pozos proyectados (PP): 2

- Cámaras reductoras de presión (CRP): 5

- Cámaras de ingreso (CI): 1

TABLA 2. DESCRIPCIÓN DE LAS ESTACIONES DEL SUBSISTEMA N 1, 2017.

\begin{tabular}{cclcc}
\hline ÍTEM & ESTACIÓN & \multicolumn{1}{c}{ TIPO } & CARACT. & COMENTARIO \\
\hline 1 & RP-01 & Reservorio & Proyectada & Tendrá cerco perimétrico y torre ventada. \\
\hline 2 & RP-02 & Reservorio & Proyectada & Tendrá cerco perimétrico y torre ventada. \\
\hline 3 & PP-01 & Pozo & Proyectada & Tendrá cerco perimétrico y torre ventada. \\
\hline 4 & PP-02 & Pozo & Proyectada & Tendrá cerco perimétrico y torre ventada. \\
\hline 5 & CRP-01 & $\begin{array}{l}\text { Cámara reductora } \\
\text { de presión }\end{array}$ & Proyectada & No tendrá cerco perimétrico. Tendrá poste. \\
\hline
\end{tabular}




\begin{tabular}{cclll}
\hline 6 & CRP-02 & $\begin{array}{l}\text { Cámara reductora } \\
\text { de presión }\end{array}$ & Proyectada & No tendrá cerco perimétrico. Tendrá poste. \\
\hline 7 & CRP-03 & $\begin{array}{l}\text { Cámara reductora } \\
\text { de presión }\end{array}$ & Proyectada & No tendrá cerco perimétrico. Tendrá poste. \\
\hline 8 & CRP-04 & $\begin{array}{l}\text { Cámara reductora } \\
\text { de presión }\end{array}$ & Proyectada & No tendrá cerco perimétrico. Tendrá poste. \\
\hline 10 & CRP-05 & $\begin{array}{l}\text { Cámara reductora } \\
\text { de presión }\end{array}$ & Proyectada & No tendrá cerco perimétrico. Tendrá poste. \\
\hline
\end{tabular}

El subsistema $\mathrm{N}^{\circ}$ 2: contiene cinco estaciones, de las cuales dos son existentes. [11]

- Reservorio existente (RE): 1

- Pozo proyectado (PP): 1

- Pozo existente (PE): 1

- Cámara reductoras de presión (CRP): 1

- Cámara de ingreso (CI): 1

TABLA 3. DESCRIPCIÓN DE LAS ESTACIONES DEL SUBSISTEMA № 2, 2017.

\begin{tabular}{ccccc}
\hline ÍTEM & ESTACIÓN & TIPO & CARACT. & COMENTARI0 \\
\hline 1 & RE-01 & Reservorio & Existente & Tendrá cerco perimétrico y torre ventada. \\
\hline 2 & PP-02 & Pozo & Proyectada & Tendrá cerco perimétrico y torre ventada. \\
\hline 3 & PE- 773 & Pozo & Existente & Tendrá cerco perimétrico y torre ventada. \\
\hline 4 & CRP- 06 & $\begin{array}{c}\text { Cámara reductora } \\
\text { de presión }\end{array}$ & Proyectada & No tendrá cerco perimétrico. Tendrá Poste. \\
\hline 5 & Cl-02 & $\begin{array}{c}\text { Cámara de } \\
\text { ingreso }\end{array}$ & Proyectada & No tiene cerco perimétrico. Tendrá poste. \\
\hline
\end{tabular}

El subsistema $\mathrm{N}^{\circ}$ 3: contiene diez estaciones.

- Reservorios proyectados (RP): 2

- Pozos proyectados (PP): 3

- Cámaras reductoras de presión (CRP): 3

- Cámaras de ingreso (CI): 2

TABLA 4. DESCRIPCIÓN DE LAS ESTACIONES DEL SUBSISTEMA № 3, 2017.

\begin{tabular}{|c|c|c|c|c|}
\hline ÍTEM & ESTACIÓN & TIPO & CARACT. & COMENTARIO \\
\hline 1 & RP- 04 & Reservorio & Proyectado & Tendrá cerco perimétrico y torre ventada. \\
\hline 2 & RP- 03 & Reservorio & Proyectado & Tendrá cerco perimétrico y torre ventada. \\
\hline 3 & PP- 04 & Pozo & Proyectada & Tendrá cerco perimétrico y torre ventada. \\
\hline 4 & PP- 05 & Pozo & Proyectado & Tendrá cerco perimétrico y torre ventada. \\
\hline 5 & PP- 06 & Pozo & Proyectado & Tendrá cerco perimétrico y torre ventada. \\
\hline
\end{tabular}




\begin{tabular}{ccccc}
\hline 6 & CRP-07 & $\begin{array}{c}\text { Cámara reductora } \\
\text { de presión }\end{array}$ & Proyectada & No tendrá cerco perimétrico. Tendrá poste. \\
\hline 7 & CRP-08 & $\begin{array}{r}\text { Cámara reductora } \\
\text { de presión }\end{array}$ & Proyectada & No tendrá cerco perimétrico. Tendrá poste. \\
\hline 8 & CRP-09 & $\begin{array}{r}\text { Cámara reductora } \\
\text { de presión }\end{array}$ & Proyectada & No tendrá cerco perimétrico. Tendrá poste. \\
\hline 9 & Cl-03 & $\begin{array}{c}\text { Cámara de } \\
\text { ingreso }\end{array}$ & Proyectada & No tendrá cerco perimétrico. Tendrá poste. \\
\hline 10 & Cl-04 & $\begin{array}{c}\text { Cámara de } \\
\text { ingreso }\end{array}$ & Proyectada & No tendrá cerco perimétrico. Tendrá poste. \\
\hline
\end{tabular}

El subsistema $\mathrm{N}^{\circ}$ 4: contiene diez estaciones.

- Reservorios proyectados (RP): 2

- Pozos proyectados (PP): 2

- Cámaras reductoras de presión (CRP): 4

- Cámaras de ingreso (CI): 1

- Cisterna proyectada (CP): 1

TABLA 5. DESCRIPCIÓN DE LAS ESTACIONES DEL SUBSISTEMA № 4, 2017.

\begin{tabular}{ccccc}
\hline ÍTEM & ESTACIÓN & TIP0 & CARACT. & COMENTARI0 \\
\hline 1 & RP-06 & Reservorio & Proyectado & Tendrá cerco perimétrico y torre ventada. \\
\hline 2 & RP-05 & Reservorio & Proyectado & Tendrá cerco perimétrico y torre ventada. \\
\hline 3 & PP-10 & Pozo & Proyectada & Tendrá cerco perimétrico y torre ventada. \\
\hline 4 & PP-11 & Pozo & Proyectado & Tendrá cerco perimétrico y torre ventada. \\
\hline 5 & CRP-10 & $\begin{array}{c}\text { Cámara reductora de } \\
\text { presión }\end{array}$ & Proyectada & No tendrá cerco perimétrico. Tendrá poste. \\
\hline 7 & CRP-11 & $\begin{array}{c}\text { Cámara reductora de } \\
\text { presión }\end{array}$ & Proyectada & No tendrá cerco perimétrico. Tendrá poste. \\
\hline 8 & CRP - 13 & $\begin{array}{c}\text { Cámara reductora de } \\
\text { presión }\end{array}$ & Proyectada & No tendrá cerco perimétrico. Tendrá poste. \\
\hline 9 & Cl-05 & Cámara de ingreso & Proyectada & No tendrá cerco perimétrico. Tendrá poste. \\
\hline 10 & CP-01 & Cisterna & Proyectada & Tendrá cerco perimétrico y torre ventada. \\
\hline
\end{tabular}

\section{DISEÑO DEL SISTEMA DE COMUNICACIÓN LOCAL (SCL) ENTRE ESTACIONES}

El SCL está conformado por los enlaces de radio entre estaciones remotas. A continuación, se describen los enlaces, cálculos de ganancias de antena y altura de las torres ventadas o postes. En las tablas siguientes, se visualizan los enlaces a implementar. 
TABLA 6. ENLACES DEL SCL - SUBSISTEMA N 1, 2017.

\begin{tabular}{|c|c|c|c|}
\hline \multirow{2}{*}{ ÍTEM } & \multicolumn{2}{|c|}{ ENLACE } & \multirow{2}{*}{ TIPO DE ENLACE } \\
\hline & ESTACIÓN 1 & ESTACIÓN 2 & \\
\hline 1 & CRP-01 & CRP-02 & RADIO \\
\hline 2 & CRP-03 & CRP-04 & RADIO \\
\hline 3 & CRP-04 & CRP-05 & RADIO \\
\hline 4 & CRP-04 & PP-01 & RADIO \\
\hline 5 & CRP-05 & PP-02 & RADIO \\
\hline 6 & RP-01 & Cl-01 & PAR TRENZADO \\
\hline 7 & RP-01 & CRP-01 & RADIO \\
\hline 8 & CRP-02 & CRP-03 & FIBRA ÓPTICA \\
\hline 9 & (RP-02 & $\mathrm{RP}-02$ & RADIO \\
\hline
\end{tabular}

TABLA 7. ENLACES DEL SCL - SUBSISTEMA №2, 2017.

\begin{tabular}{|c|c|c|c|}
\hline \multirow{2}{*}{ ITEM } & \multicolumn{2}{|c|}{ ENLACE } & \multirow{2}{*}{ TIPO DE ENLACE } \\
\hline & ESTACIÓN 1 & ESTACIÓN 2 & \\
\hline 1 & PP-03 & RE-01 & RADIO \\
\hline 2 & RE-01 & CRP-06 & RADIO \\
\hline 3 & RE-01 & Cl-02 & RADIO \\
\hline 4 & RE-01 & PE-773 & RADIO \\
\hline
\end{tabular}

TABLA 8. ENLACES DEL SCL - SUBSISTEMA N³. ELABORACIÓN PROPIA, 2017.

\begin{tabular}{|c|c|c|c|}
\hline \multirow{2}{*}{ ÍTEM } & \multicolumn{2}{|c|}{ ENLACE } & \multirow{2}{*}{ TIPO DE ENLACE } \\
\hline & ESTACIÓN 1 & ESTACIÓN 2 & \\
\hline 1 & CRP-08 & CRP-09 & RADI0 \\
\hline 2 & PP-04 & PP-05 & RADIO \\
\hline 3 & PP-05 & $\mathrm{Cl}-03$ & RADIO \\
\hline 4 & PP-05 & PP-06 & RADIO \\
\hline 5 & RP-03 & RP-04 & RADIO \\
\hline 6 & RP-04 & CRP-08 & RADIO \\
\hline 7 & RP-04 & PP-06 & RADIO \\
\hline 8 & RP-04 & $\mathrm{Cl}-04$ & RADIO \\
\hline 9 & RP-03 & CRP-07 & RADIO \\
\hline
\end{tabular}


TABLA 9. ENLACES DEL SCL - SUBSISTEMA № 4, 2017.

\begin{tabular}{|c|c|c|c|}
\hline \multirow{2}{*}{ ITEM } & \multicolumn{2}{|c|}{ ENLACE } & \multirow{2}{*}{ TIPO DE ENLACE } \\
\hline & ESTACION 1 & ESTACION 2 & \\
\hline 1 & CRP-10 & RP-06 & RADIO \\
\hline 2 & CRP-12 & CRP-13 & RADIO \\
\hline 3 & CRP-13 & CP-01 & RADIO \\
\hline 4 & CRP-13 & PP-10 & RADIO \\
\hline 5 & CRP-13 & PP-11 & RADIO \\
\hline 6 & RP-06 & Cl-05 & RADIO \\
\hline 7 & RP-06 & CRP-11 & RADIO \\
\hline 8 & RP-06 & CRP-12 & RADIO \\
\hline 9 & RP-06 & RP-05 & RADIO \\
\hline
\end{tabular}

\section{DISEÑO DE LA TOPOLOGÍA DE COMUNICACIONES Y CÁLCULOS DE TORRES Y POSTES}

A continuación, se realizó el análisis de los enlaces de radio, que está relacionado con la altura de las torres o postes. Para decidir de forma idónea el enlace de radio, se tomarán en cuenta:

a. Las curvas de nivel de la zona;

b. La zona habitada (calles, manzanas, parques, etc.).

El criterio de aceptación será garantizar que la primera zona de Fresnel se encuentre libre de obstáculos. Asimismo, se consideran márgenes de seguridad entre el primer radio de Fresnel y el nivel de terreno.

\section{Análisis de alturas de torres ventadas:}

Para calcular la altura de las torres ventadas, usaremos la siguiente fórmula:

$$
\text { Altura torre ventada }=\text { altura de torre }- \text { altura caseta } \ldots . . .(1)
$$

Para esa fórmula, la altura de la caseta, en promedio, será de 2,4 metros.

En el caso de las estaciones reductoras de presión, se utilizarán postes de concreto, los cuales irán enterrados según la fórmula:

$$
\text { Altura enterrada }=10 \% \text { (altura de poste })+50 \mathrm{~cm}
$$

\section{Análisis de radiopropagación:}

Por medio de esta operación, se obtendrán la ganancia de antenas, la potencia de recepción, el margen de seguridad y el ancho de banda del enlace de radio. Para ello, se utilizarán las fórmulas: 
La sensibilidad de recepción utilizada es de-97 dBm @ 1.0 Mbps

$$
\text { RSL }=\text { Pot.TX }- \text { Lcable }- \text { Lconectores + GTX }- \text { Lesp + GRX - Lcable.RX .....(4) }
$$

Pot.TX: $30 \mathrm{dBm}$.

Lcable: pérdida por cable $(\mathrm{dB})$.

Lconectores: pérdida por conectores $(\mathrm{dB})$.

G: ganancia de antena $(\mathrm{dB})$

Lesp: pérdida de espacio libre $(\mathrm{dB})$.

\section{RESULTADOS OBTENIDOS}

Para cada uno de los radioenlaces mencionados en cuadros anteriores, se procedió a realizar el siguiente procedimiento con la finalidad de determinar de qué manera los factores de transmisión intervienen en la factibilidad del sistema de comunicación:

Enlace CRP- 01 a CRP- 02

Del plano de las curvas de nivel entre estas estaciones se obtienen los datos de altura y altitud de los puntos de análisis, los mismos que se reflejan en la tabla 10 .

TABLA 10. ALTURA DE TORRES, 2017.

\begin{tabular}{ccc}
\hline ALTURA CRP-01 015 & $\mathrm{~m}$ \\
\hline ALTITUD PUNT0 1 & 15 & $\mathrm{msnm}$ \\
\hline ALTURA CRP-02 & 508 & $\mathrm{~m}$ \\
\hline ALTITUD PUNT0 2 & 15 & $\mathrm{msnm}$ \\
\hline
\end{tabular}

\section{Análisis de altura de poste:}

La cámara reductora de presión CRP-01 y CRP-02 no cuenta con cerco perimétrico, por lo que se tendrá que instalar un poste de $15 \mathrm{~m}$. Este contará con un pastoral con mástil, donde se montará la antena y se obtendrán los siguientes datos:

TABLA 11. ALTURA DEL POSTE. ELABORACIÓN PROPIA, 2017.

\begin{tabular}{cc}
\hline ESTACIÓN & ALTURA POSTES \\
\hline CRP-01 01 poste (15 metros) \\
\hline CRP-02 01 poste (15 metros) \\
\hline
\end{tabular}

En la siguiente figura, se visualiza el estudio de la línea de vista y la zona de Fresnel. Asimismo, se analizan las alturas máximas de los obstáculos que no van a perjudicar el enlace de radio. 


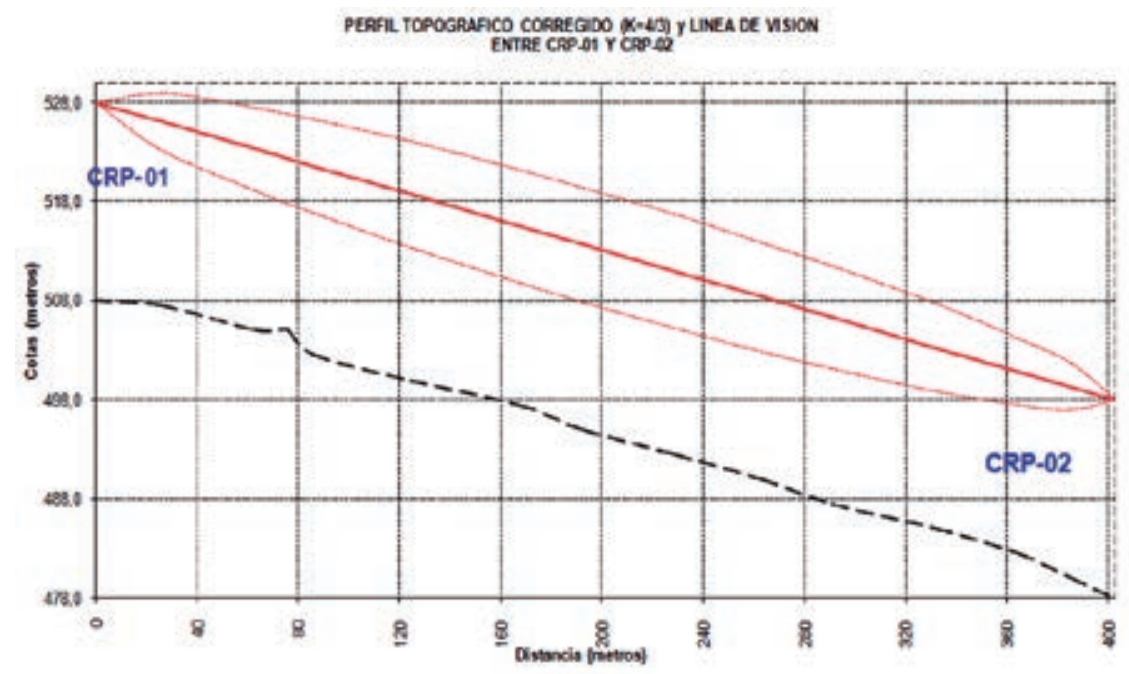

Figura 1. Perfil topográfico CRP-01 Y CRP-02. Elaboración propia, 2017.

\section{Análisis de la factibilidad de radiopropagación:}

En el cuadro $\mathrm{N}^{\circ} 12$, se visualizan los cálculos de Radiopropagación. Con ello, se concluye que el enlace será satisfactorio con un amplio margen de seguridad (55.09 dB) y un ancho de banda teórico de 1 Mbps.

TABLA 12. CÁLCULOS DE RADIOPROPAGACIÓN. (2017)

\begin{tabular}{|c|c|c|}
\hline \multicolumn{3}{|c|}{ DATOS DEL ENLACE } \\
\hline Frecuencia de operación & \multicolumn{2}{|c|}{$902-928 \mathrm{MHz}$} \\
\hline Modulación & \multicolumn{2}{|c|}{ FHSS } \\
\hline Distancia & 0,40283 & $\mathrm{Km}$ \\
\hline \multicolumn{3}{|c|}{ DATOS DEL EQUIPO } \\
\hline Potencia Tx & 28 & $\mathrm{~dB}$ \\
\hline Sensibilidad Rx @ 1 Mbps & -97 & $d B$ \\
\hline \multicolumn{3}{|c|}{ CABLE } \\
\hline Atenuación de cable & 0,1345 & $\mathrm{~dB} / \mathrm{m}$ \\
\hline Distancia de cable (TX) & 23 & M \\
\hline Distancia de cable (RX) & 23 & M \\
\hline \multicolumn{3}{|c|}{ ANTENA } \\
\hline TIPO & \multicolumn{2}{|c|}{ YAGI } \\
\hline Ganancia antena (TX) & 11 & $\mathrm{~dB}$ \\
\hline Ganancia antena (RX) & 11 & $d B$ \\
\hline \multicolumn{3}{|c|}{ PÉRDIDAS } \\
\hline Pérdida de cable (TX) & 3,09 & $d B$ \\
\hline Pérdida de cable (RX) & 3,09 & $d B$ \\
\hline Pérdida espacio libre & 83,73 & $d B$ \\
\hline
\end{tabular}




\begin{tabular}{ccc}
\hline Pérdida por pigtail & 2 & $\mathrm{~dB}$ \\
\hline Otros (vegetación, ...) & 0 & $\mathrm{~dB}$ \\
\hline & CÁLCULOS & \\
\hline PIRE (TX) & 34,9065 & $\mathrm{~dB}$ \\
\hline PIRE (RX) & 34,9065 & $\mathrm{~dB}$ \\
\hline RSL & $-41,91$ & $\mathrm{dBm}$ \\
\hline MARGEN DE SEGURIDAD (M) & \\
\hline Margen de seguridad (M) & 55,09 & $\mathrm{~dB}$ \\
\hline SNR & 63,09 & $\mathrm{~dB}$ \\
\hline
\end{tabular}

En este caso, regulamos la potencia de transmisión para no sobrepasar el PIRE de 36 dB (regulado por el MTC). En conclusión, el enlace es factible.

\section{ESQUEMA FINAL DEL SISTEMA DE MONITOREO}

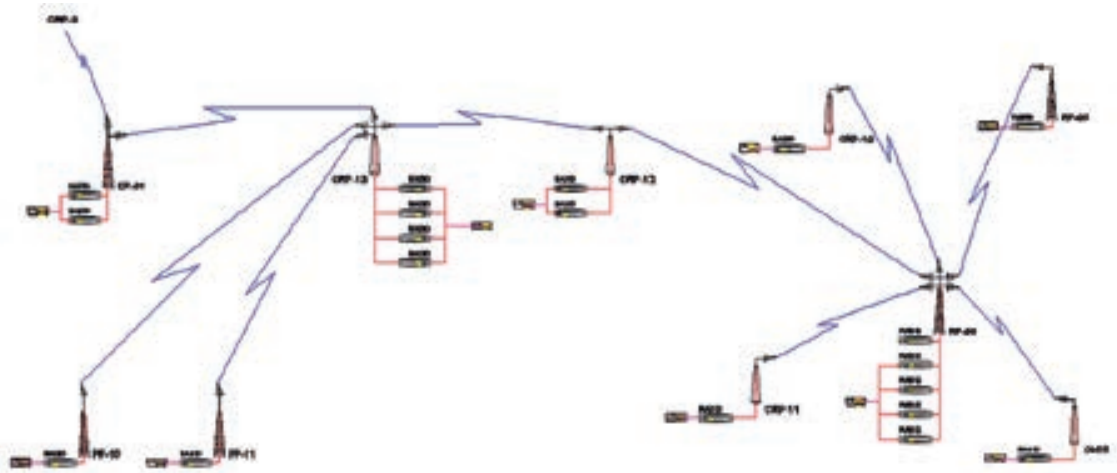

Figura 2. Esquema del subsistema hidrico N² 2. Elaboración propia, 2017.

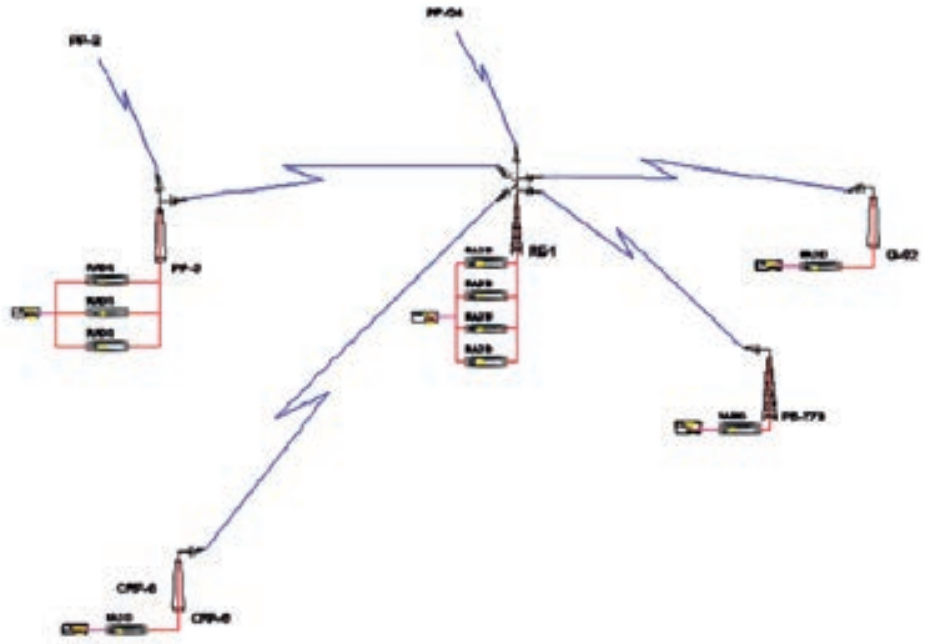

Figura 3. Esquema Sub Sistema Hidrico N 3. Elaboración propia, 2017. 


\section{CONCLUSIONES}

Se concluye que los factores del sistema de comunicación intervienen, en gran medida, en los parámetros de calidad del sistema de monitoreo. Ello permite garantizar una comunicación confiable entre las estaciones de agua potable. Así mismo, el análisis nos permite arribar a algunas conclusiones específicas, que se establecen a continuación.

Las antenas intervienen en la confiabilidad del sistema de monitoreo mediante su ganancia. Es decir, se llegó a determinar que, a mayor ganancia de la antena, crece también el valor del margen de desvanecimiento de los radioenlaces de agua potable en la población del distrito de Cieneguilla.

De igual forma, se concluye que los transmisores influyen en la confiabilidad mediante su potencia. En otras palabras, se puede asegurar que, mientras la potencia de transmisión sea mayor, lo será el valor del margen de desvanecimiento de los radioenlaces de agua potable en la población del distrito de Cieneguilla.

En todos los radioenlaces, para determinar su grado de confiabilidad, se comparó con métricas establecidas por el MTC y diversos organismos internacionales.

\section{REFERENCIAS BIBLIOGRÁFICAS}

[1] E. Cabrera, El Suministro de Agua Urbano en España. Madrid, España: Reverte, 2004.

[2] J. García, Problemática de los Abastecimientos Urbanos. Madrid, España: Universidad Politécnica de Valencia, 2005.

[3] Organización Mundial de la Salud (OMS), Guias para la calidad de agua potable. Lima, Perú: Ministerio de Salud, 2004.

[4] L. Ruiz, "Ahorro de agua y energía en el consumo sanitario», en Guía sobre Hidroeficiencia Energética. Madrid, España: Hostemur, 2012.

[5] A. Esteban, Eficiencia del agua en las ciudades. México: CECSA, 2005.

[6] UNICEF, Agua y saneamiento en el Perú. Lima, Perú, 2005.

[7] Ministerio de Vivienda Construcción y Saneamiento del Perú, Plan Nacional de Saneamiento. Lima, Perú, 2006.

[8] S. Caprile, Desarrollo de Aplicaciones con Comunicaciones Remotas. México: GAE, 2009.

[9] R. Blake, Sistemas Electrónicos de Comunicaciones. USA: Thompson, 2004.

[10] M. Apaza, Redes de Abastecimiento de Agua Potable. Madrid, España: Reverte, 2004.

[11] SEDAPAL, "Proyectos de agua potable y alcantarillado", 2017. [En línea]. Disponible en: http://www.sedapal.com.pe/proyectos-y-obras. [Accedido: 06-jul-2017]

[12] SEDAPAL, «Expedientes técnicos», 2017. [En línea]. Disponible en: http://www.sedapal.com. pe/expedientes-tecnicos. [Accedido: 05-jul-2017] 